\title{
Prevalência de adoção intra e extrafamiliar em amostras clínica e não-clínica de crianças e adolescentes
}

\author{
Prevalence of intrafamilial and extrafamilial adoption in a clinical and \\ non-clinical setting samples of children and adolescents
}

Lee Fu I ${ }^{\mathrm{a}, \mathrm{b}}$ e Eneida B Matarazzo

${ }^{a}$ Departamento de Psiquiatria da Faculdade de Medicina da Universidade de São Paulo (FM/USP). b'Ambulatório do Serviço de Psiquiatria da Infância e da Adolescência do Instituto de Psiquiatria do Hospital das Clínicas da FM/USP. São Paulo, SP, Brasil

Resumo Introdução/Objetivos: Estudos realizados em diferentes países demonstram que a proporção de crianças e adolescentes adotivos atendidos em clínicas psiquiátricas é maior em relação à verificada nas demais clínicas e na população em geral. O objetivo deste estudo é verificar a prevalência de adoção em amostra clínica e não-clínica, identificando possíveis associações entre tipos de adoção (extrafamiliar e intrafamiliar) e procura de serviço psiquiátrico.

Métodos: Estudo de corte transversal, envolvendo crianças de seis a 14 anos: amostra clínica (G1) de um serviço de psiquiatria da infância e da adolescência de uma região da cidade de São Paulo (n=551) e amostra não-clínica (G2) de uma escola localizada na mesma região ( $\mathrm{n}=365)$.

Resultados: A proporção de meninos foi significantemente maior no G1 que no G2 (p<0,001). Observou-se maior prevalência de adoção no G1 $(7,4 \%)$ que no G2 $(4,1 \%$; p=0,048). Entre as crianças adotivas, predominaram a adoção extrafamiliar, na amostra clínica $(73,2 \%)$, e a intrafamiliar, na amostra não-clínica $(60,0 \%$; p =0,030). Entre os meninos adotivos $(\mathrm{n}=32)$, também predominaram a adoção extrafamiliar no G1 $(80,8 \%)$ e a intrafamiliar no G2 $(66,7 \% ; p=0,038)$. Entre as meninas adotivas $(n=24)$, não foram constatadas diferenças entre os grupos G1 e G2 quanto à proporção de adoção extrafamiliar e intrafamiliar ( $\mathrm{p}=0,675)$.

Conclusões: Os dados sugerem que: (1) crianças adotivas são freqüentemente levadas a serviços de saúde mental; (2) a adoção intrafamiliar é a mais comum na população geral, porém são as crianças adotivas extrafamiliares que mais procuram as clínicas psiquiátricas; e (3) meninos em geral e meninos adotivos extrafamiliares são mais freqüentemente levados a serviços de saúde mental que seus pares do sexo feminino.

Descritores Adoção. Psiquiatria infantil. Criança. Adolescente.

Abstract Introduction/Objectives: Researchers from different countries have reported a higher percentage of adopted children seen in psychiatric clinics when compared to other clinics and to the general population. The objective of this study was to verify the prevalence of adopted children in two different setting samples, a clinical and nonclinical one, identifying possible associations between the type of adoption (extrafamilial or intrafamilial) and psychiatric assistance.

Methods: A cross-sectional study was carried out involving two groups of children aged six to 14 years: a clinical sample (G1) drawn from psychiatry service for children and adolescents located in a specific region of the city of São Paulo (N=551), and a non-clinical sample (G2) drawn from a school located in the same region ( $N=365)$.

Results: There was a greater proportion of boys in G1 (70\%) than in G2 $(45 \% ; \mathrm{p}<0.001)$. It was observed a greater prevalence of adoption in $\mathrm{G} 1(7.4 \%)$ when compared to $\mathrm{G} 2(4.1 \% ; \mathrm{p}=0.048)$. Among adopted children, extrafamilial adoption predominated in G1 (73.2\%), and intrafamilial adoption predominated in G2 (60.0\%; $p=0.030)$. Among adopted boys $(\mathrm{N}=32)$, there was still a predominance of extrafamilial adoption in $\mathrm{G} 1(80,8 \%)$ and still a predominance of intrafamilial adoption in $\mathrm{G} 2(66.7 \%$; $\mathrm{p}=0.038)$. Among adopted girls $(\mathrm{N}=24)$, there was no difference between the two groups regarding the proportion of extrafamilial and intrafamilial adoption $(\mathrm{p}=0.675)$.

Conclusions: These data suggest that: (1) adopted children are often taken to mental health clinics; (2) intrafamilial

Trabalho realizado na Escola Estadual de Primeiro Grau "Prof. José Cândido de Souza" e no Ambulatório do Serviço de Psiquiatria da Infância e da Adolescência do Instituto de Psiquiatria do Hospital das Clínicas da Faculdade de Medicina da Universidade de São Paulo. 
adoption is more common in the general population, but extrafamilial adopted children are the ones for whom psychiatric assistance is sought; and (3) boys as a whole and the extrafamilial adopted one are more often taken to mental health clinics when compared to girls.

\section{Keywords Adoption. Child psychiatry. Child. Adolescent.}

\section{Introdução}

Nos últimos 30 anos, vários pesquisadores constataram que a proporção de crianças e de adolescentes adotivos atendidos nos serviços de saúde mental é pelo menos duas vezes maior que a verificada na população geral. ${ }^{1-6}$ Essa constatação sugere que, mesmo sendo benéfica à maioria das crianças e dos adolescentes adotivos, a condição de viver num lar substituto parece, de alguma forma, aumentar a possibilidade de desenvolvimento de conflitos psicológicos. Essa vulnerabilidade, porém, não está associada aos transtornos psiquiátricos apresentados por adotivos na fase adulta. ${ }^{7}$

Apesar de alguns pesquisadores considerarem que a psicopatologia da criança adotada não apresenta nada de específico, ${ }^{8,9}$ Steinhauer ${ }^{10}$ entende que uma criança, por ser adotada e por ter herança genética diferente da dos outros membros de sua família adotiva, tem uma condição "especial" que a torna um alvo particularmente vulnerável. Principalmente em momentos de estresse aumentado dos pais adotivas, há uma chance maior dela ser considerada causadora de todos os males e de ser alvo de rejeição. A tendência atual é considerar a adoção um fator de proteção para reduzir a incidência de patologias psiquiátricas transmitidas geneticamente, mas que pode ser também um fator de risco quando o meio adotivo é inadequado., ${ }^{9,11}$

A adoção de crianças e adolescentes no Brasil é regulamentada pelo Estatuto da Criança e do Adolescente ${ }^{12,13}$ e é de caráter irrevogável. Pelas características socioculturais do país, a grande maioria das famílias que adotaram uma ou mais crianças não faz distinção nítida entre um processo legal de adoção e o que chamam de "filho de criação", ${ }^{14,15}$ que significa adoção sem registro judicial. A prática de adoção sem registro judicial é um procedimento comum no Brasil, e tem-se, então, dificuldade de reconhecimento da dimensão exata desse tipo de prática e também de controle e assistência.

Paralela à pouca distinção entre adoção e "criação", existem também outras variações do mesmo processo. A adoção é extrafamiliar quando a criança é adotada por pessoas que não têm relação de parentesco com nenhum de seus pais biológicos e é intrafamiliar quando a criança está sob cuidados de pessoas que têm relação de parentesco com pelo menos um dos pais biológicos. ${ }^{6} \mathrm{~A}$ distinção de vários tipos de adoção em relação aos adotantes é freqüentemente citada nos estudos, mas raramente foi investigada a influência dessas variações no desenvolvimento psicológico dos adotivos.

Por falta de estudos que abordem esse tema de forma objetiva, ainda se desconhece a proporção de adoções intrafamiliares e extrafamiliares entre os adotivos do Brasil.
Apesar da relevância das questões apresentadas, são escassos estudos sobre prevalência de adoção na população geral e na clientela de serviços de saúde mental, sendo mais raros os que procuram diferenciar adoção intrafamiliar da extrafamiliar, o que motivou a realização do presente trabalho.

\section{Objetivos}

O presente trabalho objetiva:

- verificar se a prevalência de adoção em uma amostra clínica de serviço psiquiátrico para crianças e adolescentes difere da prevalência observada em uma amostra da população não-clínica de mesma faixa etária;

- verificar se existe associação entre tipos de adoção, extrafamiliar e intrafamiliar, e procura de serviço psiquiátrico na infância e na adolescência.

\section{Métodos}

\section{Desenho do estudo}

\section{Corte transversal}

\section{Locais onde foi realizada a pesquisa}

O primeiro local foi o ambulatório do Serviço de Psiquiatria da Infância e da Adolescência (Sepia), uma unidade da coordenadoria de atendimento especializado do Instituto de Psiquiatria do Hospital das Clínicas da Faculdade de Medicina da Universidade de São Paulo (IPq-HC-FM/USP), que oferece atendimento gratuito à população das classes socioeconômicas média e baixa e que tem como norma o atendimento de pacientes até o limite máximo de 18 anos. Desde 1995, são atendidos, preferencialmente, pacientes originários de localidades situadas dentro dos limites de regionalização de atendimento do Hospital das Clínicas - de acordo com o mapa de regionalização do Sistema Único de Saúde (SUS).

No primeiro contato, pelas normas de atendimento do serviço, as crianças e os adolescentes passam por uma consulta breve de triagem para que se verifique a necessidade real de um atendimento psiquiátrico. Nessa consulta de triagem, não é regra do serviço o questionamento sobre a condição do paciente ser ou não adotivo. Se a criança ou o adolescente necessitar de assistência psiquiátrica, o caso é encaminhado para que seja marcada a primeira consulta regular. Todas as consultas realizadas no Sepia, inclusive as de triagem, são registradas pelo Serviço de Registro do IPq, e os registros são enviados ao Serviço de Arquivo Médico do IPq e, posteriormente, processados na Companhia de Processamento de 
Dados do Estado de São Paulo (Prodesp), para cômputo geral dos atendimentos realizados.

O segundo local de realização da pesquisa foi uma escola estadual situada dentro dos limites de regionalização de atendimento do Hospital das Clínicas. Foi solicitada autorização à 12a $13^{\mathrm{a}}$ e $14^{\mathrm{a}}$ Delegacias de Ensino do Estado de São Paulo (as responsáveis pela região) para a realização do presente estudo, e solicitou-se, também, uma listagem que contivesse os nomes de todas as 74 escolas públicas estaduais de primeiro e segundo graus da região; dessa listagem, foi sorteada uma escola que participou do presente estudo.

\section{Amostragem}

A amostra clínica foi constituída por um grupo de crianças e adolescentes atendidos no Sepia (G1), e a não-clínica, por alunos de uma escola pública selecionada por sorteio (G2).

\section{Amostra clínica (G1)}

A amostra clínica incluiu todas as crianças e os adolescentes de ambos os sexos, na faixa etária equivalente à da população não-clínica (G2), que apresentavam algum tipo de transtorno psiquiátrico e estavam em atendimento regular no ambulatório do Sepia no período entre $1^{\circ}$ de janeiro e 31 de dezembro de 1997. Foram incluídos todos os casos de primeiro atendimento (regular e não de triagem) durante o período mencionado e, também, os pacientes que já estavam em tratamento no Sepia antes da data determinada para o início do presente estudo e que permaneceram em atendimento por pelo menos mais três consultas regulares em 1997.

Para constituição do G1, solicitou-se à Prodesp uma listagem que contivesse todos os casos em atendimento no ambulatório do Sepia no período determinado, com as respectivas datas de nascimento. Dessa listagem, foram excluídos os pacientes que somente compareceram a uma única consulta - a de triagem - e aqueles que não estavam dentro da faixa etária equivalente à do G2. Os pacientes da listagem final constituíram o G1 do presente estudo. O banco de dado da Prodesp também forneceu informações sobre o endereço residencial dos pacientes.

\section{Amostra não-clínica (G2)}

A amostra não-clínica incluiu todas as crianças e os adolescentes de ambos os sexos, matriculados na escola selecionada no período letivo de $1^{\circ}$ de janeiro a 31 de dezembro de 1997. Para identificação dessa amostra não-clínica, solicitou-se à secretaria dessa escola uma listagem que contivesse todos os alunos matriculados no período determinado. Todos os alunos dessa listagem que concordaram em colaborar com a pesquisa vieram a constituir o grupo da amostra não-clínica (G2) do presente estudo (faixa etária de seis a 14 anos).

Após a formação dos grupos G1 e G2, o único critério de exclusão, para os dois grupos, foi domicílio de crianças e adolescentes em instituições públicas ou particulares.

Para simplificação do texto, apesar das diferentes idades, será usada a palavra crianças para referir-se a todos os sujeitos das amostras.

\section{Variáveis investigadas}

\section{Adoção}

Neste trabalho, considerou-se adotiva a criança que não vivia com ambos os pais biológicos e estava sendo criada, por período superior a um ano, por outras pessoas que, a partir de determinado momento, tinham assumido sua criação por meio ou não - de processo de adoção legal, de tutela ou de guarda.

Nesta pesquisa, a adoção foi considerada mais uma condição social e psicológica do que judicial. Valorizou-se, para a coleta de dados, a situação de uma criança que não convivia com nenhum de seus pais biológicos, e cujos cuidados diários eram de responsabilidade de outras pessoas, estabelecendo - ou não - uma relação com a criança de adoção legalizada, tutela ou guarda.

A distinção de adoção intrafamiliar e extrafamiliar foi, também, considerada nos dois grupos. Foram definidas como:

- adoção intrafamiliar (AIF), aquela em que as crianças passaram para os cuidados de algum familiar em primeiro grau (pais ou irmãos) de seus pais biológicos por um período superior a 12 meses, sendo ou não a adoção realizada por procedimento legal de adoção, tutela ou guarda.

- adoção extrafamiliar (AEF), aquela em que as crianças passaram para os cuidados de pessoas sem relação de parentesco com seus pais biológicos por um período superior a 12 meses, sendo ou não a adoção estabelecida por processo legal de adoção, tutela ou guarda.

\section{Idade}

Foi considerada a idade a ser completada pelos escolares ou pacientes no ano de 1997. Essa variável foi definida após confirmação da data de aniversário das crianças.

Como o Sepia atende pacientes de uma faixa etária mais ampla (de um a 18 anos) que a da amostra da população não-clínica, selecionada por sorteio, aguardou-se a apuração da faixa etária dos sujeitos da amostra não-clínica para determinar a faixa etária a ser adotada para a amostra clínica.

\section{Escolaridade}

Considerou-se adequada quando a criança não apresentava atraso maior que dois anos em relação à série esperada para sua idade cronológica e inadequada quando a criança apresentava um atraso maior que dois anos.

\section{Renda familiar}

Registrou-se em reais a soma dos rendimentos de cada membro da família no mês em que se realizou o levantamento dos dados, sendo a renda familiar classificada em cinco faixas: (1) até R \$ 500,00; (2) R \$ 500,00 a R \$ 1.000,00; (3) R \$ 1.000,00 a R\$ $2.000,00$; (4) R \$2.000,00 a R \$ 3.000,00; e (5) mais de R \$ 3.000,00.

\section{Instrumentos para a coleta de dados}

\section{G1}

Para coletar dados sobre a população clínica, realizou-se análise dos prontuários dos pacientes do Sepia, que con- 
têm, além da história clínica, os dados sociodemográficos e as descrições da situação sociofamiliar das crianças. A análise das condições sociofamiliares permitiu verificar se as crianças residiam ou não com seus pais biológicos, caracterizar o ambiente familiar e examinar a freqüência de contato entre pais e filhos.

\section{G2}

Para a população não-clínica, foi elaborado um questionário padronizado, em que foram solicitadas informações sobre data de nascimento, sexo, série que estava cursando e renda familiar do escolar, além de 94 perguntas objetivas sobre o estado emocional e o comportamento da criança, que permitiam apenas respostas do tipo sim ou não (Anexo).

Para a elaboração das questões que abordam o estado emocional e o comportamento, foram consultadas algumas entrevistas e escalas de diagnóstico e avaliação, e selecionadas, dentro destas, perguntas para investigação de transtornos hipercinéticos, depressão, transtornos de conduta e transtornos de ansiedade, que, segundo a literatura, são os mais prevalentes entre crianças e adolescentes. ${ }^{17}$

Os materiais consultados foram:

- Questionário Parental de Sintomas de Conners, versão de 1970, traduzido e publicado em Greehill ${ }^{18}$

- Escala de Sintomas Obsessivo-Compulsivos de Yale Brown, versão de 1986, traduzida e publicada em Jansen; ${ }^{19}$

- capítulos que abordam transtornos de ansiedade, transtornos de conduta e depressão da Entrevista Diagnóstica para Crianças e Adolescentes, versão DSM-III-R para pais, tradução do Diagnostic Interview for Children and Adolescents, DSM-III version for parents (DICA-R-P). ${ }^{20}$

Também foram incluídas, neste questionário, três perguntas com a finalidade de investigar aspectos da adoção e da "criação" por parentes: (1) mora com parentes (avós, tios ou padrinhos) e só vê os pais nos fins de semana?; (2) mora com parentes e não vê os pais há mais de um ano?; (3) foi adotado há mais de um ano por pessoas que não são familiares?

A aplicação desse questionário não teve a pretensão de detectar problemas emocionais ou de comportamento nessa amostra, mas apenas explorar e comparar a quantidade de queixas ou sintomas apresentados pelos pais das crianças adotivas e não adotivas.

Não foi investigado o aspecto étnico ("raça") dos escolares e de seus pais ou responsáveis a pedido da direção da escola, por considerar esse tipo de investigação "agressiva".

\section{Procedimentos para a coleta de dados}

Foi sorteada uma escola estadual de primeiro grau, com cursos de primeira a quarta séries do ensino fundamental. Verificou-se que havia 467 alunos matriculados, mas apenas 398 freqüentavam efetivamente as aulas. Foi preparado o mesmo número de questionários, não identificados, para os alunos efetivos. Eles foram colocados dentro de envelopes com adesivos que permitiam seu fechamento logo após serem respondidos, sendo estes distribuídos aos pais pelos professores. Na face principal do envelope, foram impressas explicações sobre a intenção de investigar o estado emocional e o comportamento dos escolares e o pedido de que devolvessem o material fechado e sem identificação, caso concordassem em participar da pesquisa. A devolução fez-se pelo depósito dos envelopes em uma urna selada, colocada em local próximo à única entrada/saída do único prédio da escola, sendo permitido acesso a essa urna apenas às autoras da pesquisa. As informações pretendidas foram coletadas de acordo com as respostas dos questionários respondidos pelos pais dos escolares.

Foram solicitados os prontuários de todos os pacientes atendidos em 1997 no Sepia, verificada a idade dos pacientes, e constituído o grupo G1 (idade equivalente à faixa etária do G2). Foram coletadas informações sobre a condição de adotivos ou não-adotivos dos integrantes do G1.

\section{Análise estatística}

Teste de qui-quadrado ${ }^{21}$ e teste exato de Fisher ${ }^{21}$ foram utilizados para comparar os grupos G1 e G2 quanto a prevalência de adoção, tipos de adoção e características demográficas. Foi considerado apenas o nível descritivo inferior a 5\% como evidência estatística de que havia diferença significante entre os grupos.

Considerando-se as cinco faixas de renda descritas na Tabela 1, o teste não-paramétrico de Mann-Whitney $\mathrm{U}$ revelou posto médio de renda mais elevado no G1 que no $\mathrm{G} 2(\mathrm{p}=0,026)$.

\section{Resultados}

Dos 398 questionários entregues aos pais do G2, foram devolvidos 371 envelopes. Excluíram-se da análise seis questionários incompletos, que, por terem apenas a primeira página respondida, não forneciam as informações pretendidas sobre adoção. Os 365 alunos que entregaram o questionário totalmente respondido $(91,7 \%)$ formaram o grupo $\mathrm{G} 2$.

Em 1997, foram atendidos no Sepia 1.000 pacientes com idade até 18 anos, sendo 47 menores de seis anos, 552 na faixa etária

Tabela 1 - Freqüência de variáveis sociodemográficas nas amostras clínica (G1) e não-clínica (G2) e níveis de significância estatística (p) para os testes do qui-quadrado.

\begin{tabular}{|c|c|c|c|}
\hline $\begin{array}{l}\text { Variáveis socio- } \\
\text { demográficas }\end{array}$ & $\begin{array}{c}\text { Sepia (G1) } \\
N(\%)\end{array}$ & $\begin{array}{c}\text { Escola (G2) } \\
N(\%)\end{array}$ & $\mathrm{p}$ \\
\hline \multicolumn{4}{|c|}{ Domicílio na região do Instituto de Psiquiatria } \\
\hline & & & $<0,001$ \\
\hline Sim & $325(59)$ & $347(95)$ & \\
\hline Não & $226(41)$ & $18(5)$ & \\
\hline \multicolumn{4}{|l|}{ Renda familiar $(\mathrm{R} \$)$} \\
\hline & & & 0,001 \\
\hline$<500$ & $165(30)$ & $146(40)$ & \\
\hline $500-1.000$ & $182(33)$ & $102(28)$ & \\
\hline $1.000-2.000$ & $127(23)$ & $62(17)$ & \\
\hline $2.000-3.000$ & $66(12)$ & $37(10)$ & \\
\hline$>3.000$ & $11(2)$ & $18(5)$ & \\
\hline \multicolumn{4}{|l|}{ Escolaridade } \\
\hline & & & 0,115 \\
\hline Adequada & $391(78)$ & $299(82)$ & \\
\hline Inadequada* & $160(22)$ & $66(10)$ & \\
\hline \multicolumn{4}{|l|}{ Sexo } \\
\hline & & & $<0,001$ \\
\hline Masculino & $386(70)$ & $164(45)$ & \\
\hline Feminino & $165(30)$ & 201 (55) & \\
\hline
\end{tabular}


de seis a 14 anos e 401 maiores de 14 anos. Dos 552 pacientes de seis a 14 anos em atendimento regular no Sepia, no período de $1^{\circ}$ de janeiro a 31 de dezembro de 1997, um tinha domicílio em instituição para menores infratores e foi excluído do estudo, sendo o G1 constituído por 551 crianças.

No G1, 325 (59\%) crianças tinham residência dentro da região de atendimento do Hospital das Clínicas delimitada pelo SUS, e, no G2, 347 (95\%) tinham domicílio na mesma região (Tabela 1). Esse resultado indicou que a proporção de crianças do $\mathrm{G} 2$ residentes na região de atendimento regionalizado foi significantemente maior que a do G1 (p<0,001).

Os grupos G1 e G2 não foram equivalentes quanto à distribuição de renda familiar, pois constatou-se diferença significante no teste do qui-quadrado ( $\mathrm{p}=0,001$; Tabela 1$)$.

Não houve diferença significante na escolarização e na adequação de escolaridade entre os dois grupos $(\mathrm{p}=0,115)$. No $\mathrm{G} 1,91 \%$ das crianças freqüentavam escola $(\mathrm{n}=501)$, sendo que $78 \%$ tinham escolaridade adequada para a idade (não mais que dois anos de atraso). No G2, 82\% das crianças apresentavam escolaridade adequada para a idade (Tabela 1).

Foram encontrados $386(70 \%)$ meninos e 165 (30\%) meninas no G1 e 164 (45\%) meninos e 201 (55\%) meninas no G2. A proporção de meninos foi significantemente maior no G1 que no G2 (p<0,001; Tabela 1).

A prevalência de adoção na amostra clínica $(7,4 \%)$ mostrouse significantemente superior à encontrada na amostra de escolares $(4,1 \%)(\mathrm{p}=0,048$; Tabela 2$)$.

Tabela 2 - Freqüência de crianças e adolescentes adotivos e não-adotivos em amostra clínica (G1) e não-clínica (G2).*

\begin{tabular}{lcccccc}
\hline Amostra & \multicolumn{2}{c}{ Adotivos } & \multicolumn{2}{c}{ Não-adotivos } & \multicolumn{2}{c}{ Total } \\
\hline & $\mathrm{N}$ & $(\%)$ & $\mathrm{N}$ & $(\%)$ & $\mathrm{N}$ & $(\%)$ \\
Sepia (G1) & 41 & $(7,4)$ & 510 & $(92,6)$ & 551 & $(100,0)$ \\
Escola (G2) & 15 & $(4,1)$ & 350 & $(95,9)$ & 365 & $(100,0)$ \\
Total & 56 & $(6,1)$ & 860 & $(93,9)$ & 916 & $(100,0)$ \\
\hline *Testa
\end{tabular}

Considerando-se apenas os adotivos nos grupos G1 e G2, observa-se que predominam a adoção extrafamiliar na amostra clínica $(73,2 \%)$ e a adoção intrafamiliar na amostra nãoclínica (60,0\%; Tabela 3). A porcentagem de adoção extrafamiliar entre os adotivos do G1 (73,2\%) foi significantemente maior que a observada entre os adotivos do

Tabela 3 - Freqüência de adoção extrafamiliar e intrafamiliar entre adotivos da amostra clínica (G1) e não-clínica (G2).*

\begin{tabular}{lcccccc}
\hline Amostra & \multicolumn{2}{c}{ Extrafamiliar } & \multicolumn{2}{c}{ Intrafamiliar } & \multicolumn{2}{c}{ Total } \\
\hline & $\mathrm{N}$ & $(\%)$ & $\mathrm{N}$ & $(\%)$ & $\mathrm{N}$ & $(\%)$ \\
Sepia (G1) & 30 & $(73,2)$ & 11 & $(26,8)$ & 41 & $(100,0)$ \\
Escola (G2) & 6 & $(40,0)$ & 9 & $(60,0)$ & 15 & $(100,0)$ \\
\hline
\end{tabular}

G2 (40,0\%) ( $\mathrm{p}=0,030$; Tabela 3).

Entre os adotivos extrafamiliares $(n=36)$, não foram constatadas diferenças entre os grupos G1 e G2 quanto à proporção de meninos e meninas $(\mathrm{p}=0,161)$. $\mathrm{O}$ mesmo ocorreu entre os adotivos intrafamiliares $(n=20) ; \mathrm{p}=1,000)$.

Entre os meninos adotivos $(\mathrm{n}=32)$, predominaram a adoção extrafamiliar no G1 $(80,8 \%)$ e a intrafamiliar no G2
$(66,7 \%)(\mathrm{p}=0,038$; Tabela 4$)$. Entre as meninas adotivas $(n=24)$, não foram constatadas diferenças entre os grupos G1 e G2 quanto à proporção de adoção extrafamiliar e

Tabela 4 - Freqüência de crianças e adolescentes do sexo masculino e feminino entre adotivos extrafamiliares e intrafamiliares em amostra clínica (G1) e não-clínica (G2).

\begin{tabular}{lrcrrrrrrr}
\hline & \multicolumn{3}{c}{ Extrafamiliares } & \multicolumn{4}{c}{ Intrafamiliares** } \\
Amostra & \multicolumn{1}{c}{ Masculino } & \multicolumn{2}{c}{ Feminino } & \multicolumn{2}{c}{ Masculino } & \multicolumn{2}{c}{ Feminino } \\
& $N$ & $(\%)$ & N & $(\%)$ & N & $(\%)$ & N & $(\%)$ \\
\hline Sepia (G1) & 21 & $(70,0)$ & 9 & $(30,0)$ & 5 & $(45,5)$ & 6 & $(54,5)$ \\
Escola (G2) & 2 & $(33,3)$ & 4 & $(66,7)$ & 4 & $(44,4)$ & 5 & $(55,6)$ \\
Total & 23 & $(63,9)$ & 13 & $(53,9)$ & 9 & $(45,0)$ & 11 & $(55,0)$ \\
\hline
\end{tabular}

*Teste exato de Fisher: $p=0,161$

${ }^{\star}$ Teste exato de Fisher: $p=1,000$

intrafamiliar ( $\mathrm{p}=0,675$; Tabela 4).

Como informação complementar, foi registrado o número de queixas que os pais apresentaram sobre os filhos e se estes recebiam ou haviam recebido algum tipo de tratamento na área de saúde mental ao longo da vida. $\mathrm{Na}$ amostra de escolares, a média do número de queixas dos pais (respostas afirmativas no questionário) permitiu verificar que os pais das crianças adotivas não assinalaram mais queixas $(27 \pm 2)$ sobre seus filhos, em comparação com os pais das crianças não-adotivas $(31 \pm 3)$. Nessa amostra, havia $45(12,3 \%)$ crianças que recebiam ou receberam algum tipo de tratamento na área de saúde mental ao longo da vida, sendo que duas eram adotivas extrafamiliares, duas eram adotivas intrafamiliares, três moravam com os avós e só encontravam os pais nos finais de semanas, e 38 não eram adotivas.

\section{Discussão}

Neste estudo, foi observado que a proporção de meninos na população clínica foi maior que a de meninas, o que sugere que, na faixa etária investigada, os meninos, incluindo-se os adotivos e os não adotivos, são mais freqüentemente levados a serviços de saúde mental que as meninas. Esse dado está de acordo com a opinião de diversos pesquisadores da área de psiquiatria da infância e da adolescência que apontam maior vulnerabilidade dos meninos aos transtornos mentais do que as meninas na idade pré-escolar e escolar. ${ }^{22}$

A maior prevalência de adoção na amostra clínica sugere que crianças e adolescentes vivendo na condição de adotivos comparecem com freqüência a serviços de psiquiatria. Algumas hipóteses podem ser aventadas para explicar esse resultado.

Algumas crianças cujos pais, por circunstâncias diversas, não tiveram condições de criá-las teriam, também, uma carga genética desfavorável, que as predisporia a problemas emocionais e de comportamento. Segundo Hersov ${ }^{9} \&$ Steinhauer, ${ }^{10}$ a maioria dos filhos adotivos origina-se de lares socioeconomicamente desfavoráveis, com pais biológicos problemáticos. Essa opinião parece bastante discutível e difícil de ser verificada, no Brasil, pela dificuldade de se obter informações sobre a maioria das famílias de origem das crianças adotadas.

O clima emocional de certos lares adotivos seria contaminado pelo excesso de intolerância e pela ansiedade de pais inférteis, preocupados em se mostrar capazes de criar seus filhos adoti- 
vos de forma satisfatória. Eles, portanto, apresentariam maior preocupação ou mais queixas sobre seus filhos adotivos, procurando com maior freqüência atendimentos nos serviços de psicologia ou psiquiatria. Schechter et $\mathrm{al}^{23}$ discordam dessa hipótese, concluindo, após um estudo em que compararam a quantidade e a qualidade das queixas apresentadas pelos pais adotivos e não-adotivos de uma população clínica, que a alta percentagem de adotivos com distúrbios emocionais nas clínicas psiquiátricas e psicológicas não parece estar relacionada à hipersensibilidade dos pais adotivos em relação às dificuldades de seus filhos. A constatação de que os pais adotivos do G2 não assinalaram mais sintomas e queixas sobre seus filhos no questionário do que os pais não-adotivos também não corroborou essa hipótese. No entanto, o pequeno número de crianças adotivas nesse grupo pode ter dificultado a identificação de possíveis diferenças entre adotivos e nãoadotivos quanto ao número de sintomas.

Como o presente estudo incluiu todas as crianças que vivem em condição de adotivas, sem distinção quanto a ter ou não passado por trâmites legais, mas diferenciando a adoção intrafamiliar (crianças criadas por familiares dos pais biológicos) da extrafamiliar, teve-se a oportunidade de examinar esse aspecto peculiar da adoção, raramente investigado na literatura.

$\mathrm{O}$ fato dos adotivos intrafamiliares terem predominado na amostra escolar sugere que esse tipo de adoção é o que predomina na população geral brasileira. No entanto, na amostra clínica, predominou a adoção extrafamiliar, sugerindo que os adotivos extrafamiliares procuram mais os serviços de saúde mental que os adotivos intrafamiliares.

Considerando-se o sexo das crianças e o tipo de adoção, observou-se que, entre os meninos adotivos da amostra clínica, predominou a adoção extrafamiliar, confirmando a hipótese de que meninos adotivos extrafamiliares procuram mais os serviços de saúde mental que os adotivos intrafamiliares. No entanto, entre as meninas adotivas tanto do G1 quanto do G2, notou-se proporção semelhante de adoção extrafamiliar e intrafamiliar, revelando que o tipo de adoção não interferiu na procura de serviços pela população de adotivos do sexo feminino.

A maior procura de serviços de saúde mental por meninos adotivos extrafamiliares coincide com os resultados de Plomin ${ }^{25}$ e Lipman et al, ${ }^{26}$ que encontraram na população geral uma probabilidade maior de meninos adotivos apresentarem algum tipo de transtorno psiquiátrico do que meninas adotivas. Como os serviços de saúde mental costumam receber mais meninos que meninas, as crianças adotivas extrafamiliares do presente estudo não diferiram das crianças em geral. Porém, os dados relativos às crianças adotivas intrafamiliares sugerem que estas procuram menos os serviços de saúde mental, e os meninos têm a mesma necessidade de assistência que as meninas.

Para explicar o fato dos adotivos intrafamiliares serem levados com menor freqüência a serviços psiquiátricos do que os extrafamiliares, duas hipóteses podem ser propostas:

1. criados por parentes, os adotivos intrafamiliares encontrariam, no lar, um ambiente mais propício a seu desenvolvimento emocional e teriam menor incidência de problemas de comportamento;
2. devido ao grau de parentesco, a família adotiva teria maior tolerância em relação a eventuais distúrbios psíquicos apresentados pelo filho e, portanto, não procurariam ajuda profissional.

O fato de a maioria dos estudos com adotivos ter sido realizada com adotivos extrafamiliares (não parentes), somado à escassez de estudos que diferenciam a adoção extrafamiliar da intrafamiliar, torna difícil avaliar as reais consequiências dos dois processos.

Levantamentos realizados na Inglaterra e no Canadá mostraram que aproximadamente $50 \%$ de todas as adoções realizadas nesses países são intrafamiliares. No entanto, é importante notar que, diferentemente da amostra do presente estudo, a maioria deles se refere à adoção de uma criança por seus padrastos ou madrastas, ${ }^{9,10}$ enquanto, no Brasil, foi mais comum encontrar crianças que tinham tios ou avós biológicos como seus responsáveis legais. O número de adoções intrafamiliares (mesma definição utilizada pelo estudo canadense) tem aumentado consideravelmente na Inglaterra e nos EUA, provavelmente apenas refletindo o aumento de taxa de divórcio e de novos casamentos..$^{8,10}$

A adoção intrafamiliar (por avós, tios e outros parentes biológicos) é considerada, por diversos autores, como sendo mais problemática por geralmente envolver situações familiares mais complexas e motivações para adoção diferentes das observadas nas adoções extrafamiliares. ${ }^{6,9,10}$ Em alguns dos casos de adoção intrafamiliar, a guarda ou a adoção de uma criança é determinada por processos judiciais, e os familiares são, na verdade, obrigados a assumir os cuidados com a criança mesmo que não estejam motivados ou preparados para isto. Em outros casos, os familiares assumem a responsabilidade sobre o filho de um parente por motivos religiosos, morais ou sentimentos de culpa inapropriados, sendo as reais motivações muitas vezes desconhecidas pelos próprios indivíduos, que podem estar semeando, assim, um campo para futuros conflitos, após a inserção da criança no lar adotivo. ${ }^{9,16}$ No presente estudo, verificou-se que quase um terço das crianças adotivas da amostra clínica eram criadas pelos avós ou tios biológicos. Essa proporção ressalta a necessidade de futuras investigações sobre a influência da adoção intrafamiliar no desenvolvimento psicológico e sobre o grau de desajustes emocionais e comportamentais naqueles que foram criados por avós ou tios biológicos, comparados aos adotivos extrafamiliares e não-adotivos.

Quanto a possíveis vieses no presente estudo, observa-se que aproximadamente $8 \%$ dos alunos da escola (33 escolares) não participaram da pesquisa. No entanto, esta não parece ser uma perda seletiva que envolvesse adotivos em número suficiente para modificar os resultados da pesquisa, pois a própria escola informou que a proporção de meninos e meninas e a proporção de alunos com atraso escolar, observadas no presente estudo, foram compatíveis com os resultados de levantamentos realizados pela secretaria da escola. Além disso, a diferença entre os grupos G1 e G2 quanto à prevalência de adoção pode ter sido influenciada por diferenças de nível socioeconômico, pois a adoção de crianças por tios e avós é mais freqüente nas classes sociais mais baixas. No entanto, como não foi possível calcular a renda familiar mensal per capita, não se pode saber ao certo se 
existe de fato uma diferença de renda entre os grupos G1 e G2.

Os trabalhos realizados no Brasil sobre o tema da adoção, presentes na literatura da área de psiquiatria e de psicologia, são escassos. A característica cultural do povo brasileiro em diferenciar pouco o procedimento legal de adoção e o de "criação", associada à despreocupação, por parte dos governantes, em investir num cadastro que inclua as adoções ilegais, e a inacessibilidade ao cadastro de processos de adoção que passaram por trâmites legais impossibilitam o conhecimento do número real da população de adotivos no Brasil, ${ }^{15}$ dificultando investigações e estudos comparativos ligados a esse tema. Somada aos entraves legais, há também uma crença popular de que filhos adotivos são sinônimos de problemas, conduzindo os profissionais da área de saúde mental à tentativa de encontrar nelas uma psicopatologia específica que confirme a tal crença, o que seria absolutamente incorreto. ${ }^{2}$

\section{Conclusão}

O presente estudo foi planejado na tentativa de explorar um tema ainda pouco investigado no Brasil. O caráter exploratório do estudo permitiu visualizar aspectos desconhecidos da população de crianças e adolescentes adotivos brasileiros.

Os dados obtidos sugerem que: (1) crianças adotivas são freqüentemente levadas a serviços de saúde mental; (2) a adoção intrafamiliar é a mais comum na população geral, porém são as crianças adotivas extrafamiliares que mais procuram as clínicas psiquiátricas; e (3) os meninos em geral e os meninos adotivos extrafamiliares são mais freqüentemente levados a serviços de saúde mental que seus pares do sexo feminino. A adoção intrafamiliar não parece estar associada à procura de atendimento em serviços de saúde mental.

O presente trabalho mostra a necessidade de futuros estudos, com amostras maiores, para verificar com maior profundidade os aspectos abordados e examinar possíveis associações entre tipos de adoção e presença de transtornos psiquiátricos em crianças e adolescentes. Seria importante partir da população geral, identificando amostras aleatórias de adotivos intrafamiliares, adotivos extrafamiliares e não-adotivos para verificar as respectivas necessidades de assistência na área de saúde mental.

\section{Referências}

1. Schechter MD. Observations on adopted children. Arch Gen Psychiat 1960;10(2):109-18.

2. Jerome DG, Silbertein RM, Mandell W. Adopted children brought to child psychiatric clinic. Arch Gen Psychiat 1966;3:526-31.

3. Bohman M. Alternatives to biological parenting: a survey of earlier and ongoing adoption research. In: Grahan PJ, editor. Epidemiological approaches in child psychiatry. $1^{\text {st }}$ ed. London: Academic Press; 1977. p. 323-31.

4. Steinberg D. Causes of disorders and Influences on development. In: Basic adolescent psychiatry. Oxford: Black-Well Scientific Publications; 1987. p. 30-49.

5. Dickson LR, Heffron WM, Parker C. Children from disrupted and adoptive homes on an impatient unit. Am J Orthopsychiat 1990;60(4):594-602.

6. Schechter MD. Adoption. In: Kaplan HI, Sadock BJ, editors. Comprehensive textbook of psychiatry. $5^{\text {th }}$ ed. Baltimore: Williams \& Wilkins; 1989. p. 1958-62.

7. Brinich PM, Brinich EB. Adoption and adaptation. J Nerv Ment Dis 1982;170(8):489-93.

8. Ajuriaguerra J, Marcelli D. A criança em sua família. In: Manual de psicopatologia infantil. Tradução de Filman AE, editor. São Paulo: Masson; 1986. p. 340-69.

9. Hersov L. The seventh Jack Tizard memorial lecture. Aspects of adoption. J Child Psychol Psychiat 1990;4:493-510.

10. Steinhauer PD. Adoption. In: Garfinkel BD, Carlson GA, Weller EB, editors. Psychiatric disorders in children and adolescents. Philadelphia: WB Sauders; 1990. p. 428-40.

11. Hersov L. Adoption. In: Rutter M, Taylor E, Hersov L, editors. Child and adolescent psychiatry: modern approaches. $3^{\text {rd }}$ ed. Oxford: Black-well Scientific; 1994. p. 267-82.

12. Secretaria da Criança e Família e Bem Estar Social São Paulo. Direitos da criança e do adolescente. São Paulo: Imprensa Oficial do Estado; 1997. p. 64.

13. Oliveira J. Do direito à convivência familiar e comunitária. Estatuto da Criança e do Adolescente. 6a ed. São Paulo: Saraiva; 1996. p. 4-9.

14. Luce C, Silva TM, Saggese EG. A mística da adoção: o adolescente e o lar substituto. J Bras Psiquiat 1983;32(3):173-86.

15. Weber LND. Família adotivas e mitos sobre laços de sangue. Teoria na prática "Caderno Técnico" n. 15. CRP- 8"; maio de 1996.

16. Derdeyn AP. Questões psicológicas na adoção. In: Lewis M, editor. Tratado de psiquiatria da infância e adolescência. Ortiz ICS, tradutor. Porto Alegre: Artes Médicas; 1995. p. 1113-21.

17. Anderson JC, Williams S, McGree R, Silva PA. DSM-3 disorders in preadolescent children: prevalence in a large sample from the general population. Arch Gen Psychiat 1987;69(1):69-76.

18. Greehill LL. Transtorno de déficit de atenção por hiperatividade em crianças. In: Transtornos psiquiátricos na infância e adolescência. Goulart MCM, tradutor. Porto Alegre: Artes Médicas; 1992. p. 135-62.

19. Jensen JB. Transtorno obsessivo-compulsivo em crianças e adolescentes. In: Transtornos psiquiátricos na infância e adolescência. Goulart MCM, tradutor. Porto Alegre: Artes Médicas; 1992. p. 82-99.

20. Reich W, Welner Z, Herjanic B. Diagnostic interview for children \& adolescents [Revised]. New York: Multi-Health Systems Inc.; 1996.

21. Agresti A. Categorical data analysis. New York: John Wiley Sons; 1990.

22. Cadoret RJ, Cain C. Sex differences in predictors of antisocial behavior's in adoptees. Arch Gen Psychiat 1980;37:1171-5.

23. Schechter MD, Carlson PV, Simmons III JQ, Work HH. Emotional problems in the adoptees. Arch Gen Psychiat 1964;10(2):109-18.

24. Versluis-Den Bieman HJ, Verhulst FC. Self-reported and parent reported problems in adolescent international adoptees. J Child Psychol Psychiat 1995;36:1411.

25. Plomin R. Genetics and children's experiences in the family. J Child Psychol Psychiat 1995;36(1):33-68.

26. Lipman EL, Offord DR, Racine YA, Boyle MH. Psychiatric disorders in adopted children: a profile from the Ontario child health study. Can J Psychiat 1992;37(9):627-33.

27. Di Loreto ODM. Da Adoção [e dos erros do pensar] ou dos erros de pensar [e da adoção]. Psicologia Estudo 1997;2(2):1-33.

\section{Correspondência}

Lee Fu I

Alameda Ministro Rocha Azevedo, 464, 91

01410-001 São Paulo, SP

Tel:(0xx11)3064-4948

E-mail: leefui@attglobal.net 